\title{
TARGETED ALLELIC SEQUENCING VALIDATION OF CONDITIONING THERAPY-INDUCED ORAL MUCOSITIS- ASSOCIATED SINGLE NUCLEOTIDE POLYMORPHISMS (SNPS) IDENTIFIED BY EXOME SEQUENCING
}

\author{
$\underline{\text { J-L. Mougeot }}^{1}$, V. Talevi ${ }^{1}$, B. Hasséus ${ }^{2}$, K. Garming-Legert ${ }^{3}$, A. Hovan ${ }^{4}$, J.E. Johansson ${ }^{5}$, I. von Bültzingslöwen ${ }^{6}$, M. Brennan ${ }^{1}$, F. Mougeot ${ }^{1}$
}

\begin{abstract}
${ }^{1}$ Carolinas Medical Center- Atrium Health, Oral Medicine, Charlotte, USA; ${ }^{2}$ Institute of Odontology- University of Gothenburg, Oral Medicine and Pathology, Gothenburg, Sweden; ${ }^{3}$ Karolinska Institute, Dental Medicine, Stockholm, Sweden; ${ }^{4}$ University of British Columbia, Radiation Oncology, Vancouver, Canada; ${ }^{5}$ Department of Hematology and Coagulation, Sahlgrenska University Hospital, Gothenburg, Sweden; ' University of Gothenburg, Department of Oral Microbiology and Immunology, Gothenburg, Sweden
\end{abstract}

\section{Abstract}

INTRODUCTION: Oral mucositis $(\mathrm{OM})$ is a common dose-limiting side effect of conditioning therapy for patients with hematologic cancer undergoing hematopoietic stem cell transplant (HSTC) [1]. Using exome sequencing in a pilot study, we have previously identified nine genes with SNPs associated with OM in HSCT patients [2].

OBJECTIVE: Using targeted allelic sequencing, our objective was to validate the candidate SNPs.

METHODS: Saliva DNA from HSCT patients $(n=63)$, that was previously analyzed by whole genome exome sequencing, was subjected to targeted allelic sequencing for nine candidate genes. Sequencing was performed by Illumina HiSeq TruSeq paired-end sequencing of PCR amplicons of the targeted regions [3]. SNPs/INDELs were identified using basic variant detection model within the CLC Genomics Server software v9.0.1.

RESULTS: Of the nine genes, LAMC1, had 8 SNP locations which were either all heterozygous or homozygous for 56 out of the 63 patients. The full homozygote variant genotype was overrepresented in patients with OM WHO score 1-4. While targeted allelic sequencing confirmed the genotypes for the 56 patients, of the 16 ambiguous SNP locations for the remaining seven patients, 11 were corrected per conserved pattern. Indeed, one patient (WHO score=1), who had 5 ambiguous SNP locations in LAMC1, was confirmed having the full homozygote variant genotype. While SNP correction was related to low DNA concentration, non-correction was associated with lower DNA quality. Overall accuracy for the 9 genes, including 14 corrections, was $99.3 \%$.

CONCLUSIONS: Targeted allelic sequencing is an effective approach for confirming select SNPs prior to engaging into large sample size investigation of SNPs associated with OM in HSCT patients.

\section{Results}

- SNPs/INDELs detection by targeted allelic sequencing for nine candidate genes

HSCT patients' saliva DNA $(n=63)$

Paired-end sequencing of PCR amplicons of the targeted regions

SNPs/INDELs detection

$$
\zeta
$$

Comparison of SNP genotypes identified by exome sequencing and targeted allelic sequencing

- Overall results:

- 14 SNP locations were corrected

- 2 SNP locations were not corrected

- 2 SNP locations were not called with the right genotype

- 3 SNP locations are still ambiguous

\begin{tabular}{|l|c|c|}
\hline & $\begin{array}{c}\text { Exome } \\
\text { sequencing }\end{array}$ & $\begin{array}{c}\text { Targeted allelic } \\
\text { sequencing }\end{array}$ \\
\hline Overall accuracy & $98.3 \%$ & $99.3 \%$
\end{tabular}

- LAMC1 has 8 SNP locations which we expect to be either all heterozygous or homozygous per conserved pattern:

- 12 SNP locations were corrected

- 2 SNP locations were not corrected

- 2 SNP locations were not called with the right genotype

\begin{tabular}{|c|c|c|}
\hline \multicolumn{2}{|c|}{ LAMC1 validation summary table } \\
\hline & $\begin{array}{c}\text { Exome } \\
\text { sequencing }\end{array}$ & $\begin{array}{c}\text { Targeted allelic } \\
\text { sequencing }\end{array}$ \\
\hline $\begin{array}{c}\text { Patients with } \\
\text { conserved pattern } \\
\text { genotype }\end{array}$ & $56 / 63$ & $61 / 63$ \\
\hline $\begin{array}{c}\text { \% of correct SNP } \\
\text { genotypes }\end{array}$ & $\begin{array}{c}97.2 \% \\
{[=(490 / 504) \times 100]}\end{array}$ & $\begin{array}{c}99.3 \% \\
{[=(500 / 504) \times 100]}\end{array}$ \\
\hline
\end{tabular}

- FAAH2 has 2 SNP locations which we expect to be either all heterozygous or homozygous per conserved pattern:

- 1 SNP location was corrected

- 1 SNP location was determined which exome sequencing was unable to identify

\section{Methods}

- PCR amplicons of the targeted nine genes using paired-end sequenced by Illumina HiSeq TrueSeq

- Comparison between exome sequencing and targeted allelic sequencing results to validate the SNPs and to identify the improvements and the weaknesses of targeted allelic sequencing
- SNPs/INDELs identified using CLC Genomics Server software v9.0.1

\section{Conclusion}

- Targeted allelic sequencing has improved the accuracy of SNPs/INDELs calling by $1 \%$.

- From the targeted allelic sequencing analysis, SNP correction was related to low DNA concentration while non-correction was associated with lower DNA quality.

\section{Acknowledgments \\ Acknowledgments}

\section{References}

1 - Bowen JM, Wardill HR. Advances in the understanding and management of mucositis during stem cell transplantation. Curren Opinion in Supportive and Palliative Care; 2017 Dec;11(4):341-346.

2 - Mougeot JL, Hasseeus B, Garming-Legert K, Rozario N, Hovan A, von Bültzingslöwen I, Brennan M, Mougeot F. Conditioning Therapy-Induced Oral Mucositis-Associated Single Nucleotide Polymorphisms (SNPs) Identified by Exome Sequencing Analysis: A Pilot Study. Support Care Cancer; 2018; 26 (Suppl 2): S148 (eP191)

- Xu C, Nezami Ranjbar MR, Wu Z, DiCarlo J, Wang Y. Detecting very low allele fraction variants using targeted DNA sequencing and a novel molecular barcode-aware variant caller. BMC Genomics. 2017;18(1):5.
This study was funded by Atrium Health Foundation / Freedland Cancer Research Fund 